\title{
THE NEW $v$-METRIC INDUCES THE CLASSICAL GAP TOPOLOGY
}

\begin{abstract}
AMOL SASANE
Abstract. Let $\mathscr{A}_{+}$denote the set of Laplace transforms of complex Borel measures $\mu$ on $[0,+\infty)$ such that $\mu$ does not have a singular non-atomic part. In [1], an extension of the classical $v$ metric of Vinnicombe was given, which allowed one to address robust stabilization problems for unstable plants over $\mathscr{A}_{+}$. In this article, we show that this new $v$-metric gives a topology on unstable plants which coincides with the classical gap topology for unstable plants over $\mathscr{A}_{+}$ with a single input and a single output.
\end{abstract}

Mathematics subject classification (2010): Primary 93B36; Secondary 93D15, 46J15. Keywords and phrases: $v$-metric, robust control, Banach algebras.

\section{REFERENCES}

[1] J. A. Ball And A. J. SASAne, Extension of the v-metric, Complex Analysis and Operator Theory, to appear.

[2] A. BRUDNy AND A. J. SASANE, Sufficient conditions for the projective freeness of Banach algebras, Journal of Functional Analysis, in press.

[3] A. BÖtTCher AND B. Silbermann, Analysis of Toeplitz operators, Springer-Verlag, Berlin, 1990.

[4] R. G. Douglas, On the $C^{*}$-algebra of a one-parameter semigroup of isometries, Acta Mathematica 128, 3-4 (1972), 143-151.

[5] R. G. Douglas, Banach algebra techniques in the theory of Toeplitz operators, Conference Board of the Mathematical Sciences Regional Conference Series in Mathematics, No. 15. American Mathematical Society, Providence, R.I., 1973.

[6] A. K. EL-SAKKARY, The gap metric: robustness of stabilization of feedback systems, IEEE Transactions on Automatic Control 30, 3 (1985), 240-247.

[7] T. T. Georgiou, On the computation of the gap metric, Systems Control Letters 11, 4 (1988), 253257.

[8] T. T. Georgiou And M. C. Smith, Optimal robustness in the gap metric, IEEE Transactions on Automatic Control 35, 6 (1990), 673-686.

[9] B. Jessen AND H. ToRnehaVe, Mean motions and zeros of almost periodic functions, Acta Mathematica 77 (1945), 137-279.

[10] K. M. Miknola, Infinite-dimensional linear systems, optimal control and algebraic Riccati equations, Doctoral dissertation, Technical Report A452, Institute of Mathematics, Helsinki University of Technology, 2002.

[11] N. K. NIKOLSKI, Treatise on the shift operator, Spectral function theory. With an appendix by S.V. Khrushchëv and V. V. Peller. Grundlehren der Mathematischen Wissenschaften 273, Springer-Verlag, Berlin, 1986.

[12] N. K. NiKOLSKI, Operators, functions, and systems: an easy reading. Volume 1. Mathematical Surveys and Monographs, 92. American Mathematical Society, Providence, RI, 2002.

[13] J. R. PARTington, Linear operators and linear systems. An analytical approach to control theory, London Mathematical Society Student Texts 60, Cambridge University Press, Cambridge, 2004.

[14] M. VIDYASAGAR, The graph metric for unstable plants and robustness estimates for feedback stability, IEEE Transactions on Automatic Control 29, 5 (1984), 403-418.

[15] G. VinnicombE, Frequency domain uncertainty and the graph topology, IEEE Transactions on Automatic Control 38, 9 (1993), 1371-1383. 
[16] G. Zames And A. K. El-SAKKary, Unstable systems and feedback: The gap metric, In Proceedings of the Allerton Conference, 380-385, Oct. 1980. 